\title{
Management of Mycoplasma genitalium infections - can we hit a moving target?
}

Jørgen Skov Jensen ${ }^{1 *}$ and Catriona Bradshaw ${ }^{2,3}$

\begin{abstract}
Mycoplasma genitalium is an etiological agent of sexually transmitted infections, but due to its fastidious growth requirements, only a few $M$. genitalium strains are available for determination of the activity of currently used and new antimicrobial agents.

Recent clinical trials have demonstrated that treatment with azithromycin has decreasing efficacy due to an increasing prevalence of macrolide resistance, which is likely to be attributed to the widespread use of $1 \mathrm{~g}$ single dose azithromycin. Second line treatment with moxifloxacin is similarly under pressure from emerging resistance. The era of single dose monotherapy for uncomplicated STIs such as M. genitalium and N. gonorrhoeae, while convenient for patients and physicians, has been associated with escalating resistance and treatment failure and is now drawing to a close. There is a critical need for trials of combinations of existing registered drugs and new antimicrobial compounds, implementation of diagnostic testing combined with molecular detection of resistance, and antimicrobial surveillance.
\end{abstract}

Keywords: Mycoplasma genitalium, Treatment, Antimicrobial resistance

\section{Review}

The importance of $M$. genitalium in genital syndromes Mycoplasma genitalium was first isolated in 1980 from two of 13 men with non-gonococcal urethritis (NGU) [1]. It is an extremely slow-growing and fastidious bacterium, and progress in determining its role as a pathogen in human disease was hampered by the lack of reliable detection methods. After the development of the first diagnostic PCRs in the early 1990's [2,3], studies on male NGU started to accumulate $[4,5]$. M. genitalium is now a wellestablished sexually transmitted infection and the etiological agent of a number of syndromes (reviewed in [6, 7]). Several studies have demonstrated the association between M. genitalium and urethritis, cervicitis, endometritis, and pelvic inflammatory disease (PID) [8-11]. In a recent metaanalysis [12], significant associations were found between M. genitalium and cervicitis (pooled odds ratio (OR) 1.66), and pelvic inflammatory disease (pooled OR 2.14). While there are less data in pregnancy, $M$. genitalium has been associated with preterm birth (pooled OR 1.89), and

\footnotetext{
* Correspondence: jsj@ssi.dk

${ }^{1}$ Microbiology and Infection Control, Sexually Transmitted Bacterial Infections, Research and Development, Statens Serum Institut, Artillerivej 5,

Copenhagen DK-2300, Denmark

Full list of author information is available at the end of the article
}

spontaneous abortion (pooled OR 1.82) [12], but the prevalence of $M$. genitalium in pregnant women has been low in some settings [13]. Serological studies and studies based on detection of $M$. genitalium using NAATs have also shown an association with increased risk of tubal factor infertility (pooled OR 2.43) [12]. In sub-analyses that accounted for co-infections, Lis et al. found these associations to be stronger and more statistically significant [12].

Several studies have demonstrated the association between M. genitalium and urethritis in men [4, 14-17] and in a meta-analysis including 37 studies up to 2010 [6], $M$. genitalium was associated with a pooled OR of 5.5 for NGU. In the 29 studies where information on chlamydial infection was available, M. genitalium was associated with a pooled OR of 7.6 for non-chlamydial non-gonococcal urethritis (NCNGU) [6]. The prevalence of $M$. genitalium in men with NCNGU ranges from $10 \%$ to $35 \%$ [6], thus contributing significantly to the overall burden of disease. In comparison, $M$. genitalium is detected in only $1 \%$ to $3.3 \%$ of men and women in the general Western European and United States population [18-20].

Signs and/or symptoms of urethritis persist in patients in which antibiotic treatment fail. In men with persistent NCNGU after doxycycline therapy, as many as $41 \%$ 
were found to be M. genitalium positive [21], and $91 \%$ of patients with persistent $M$. genitalium infection experienced persistent urethral symptoms compared to $17 \%$ of patients in whom M. genitalium was eradicated [22]. A total of 21 studies have examined the efficacy of treatment of $M$. genitalium positive urethritis, and presented data on the presence of urethritis in patients where antibiotic treatment failed to eradicate the infection [22-42]. Of the 310 patients with persistent $M$. genitalium infection, 240 (77 \%) had persistent urethritis (defined as persistent urethral symptoms and/or signs). In the 19 studies where data on both men with persistent and eradicated M. genitalium infection could be evaluated, of the 285 patients with persistent M. genitalium infection, 220
(77\%) had persistent urethritis, compared to only 78 (16\%) of the 499 patients where M. genitalium was successfully eradicated $(p<0.0001)$. Analysing the 19 studies using random effects (DerSimonian-Laird method), persistent $M$. genitalium was associated with a pooled odds ratio of 26 (95\% CI = 11 to 57 ) for persistent urethritis (signs and/or symptoms). A forest plot illustrating the odds-ratios for the individual studies is shown in Fig. 1. Two studies reported no treatment failures, and ORs could therefore not be calculated [29, 33]. This analysis clearly shows that failure to eradicate $M$. genitalium leads to persistent or recurrent signs and symptoms of urethritis in a significant proportion of men with persistent infection.

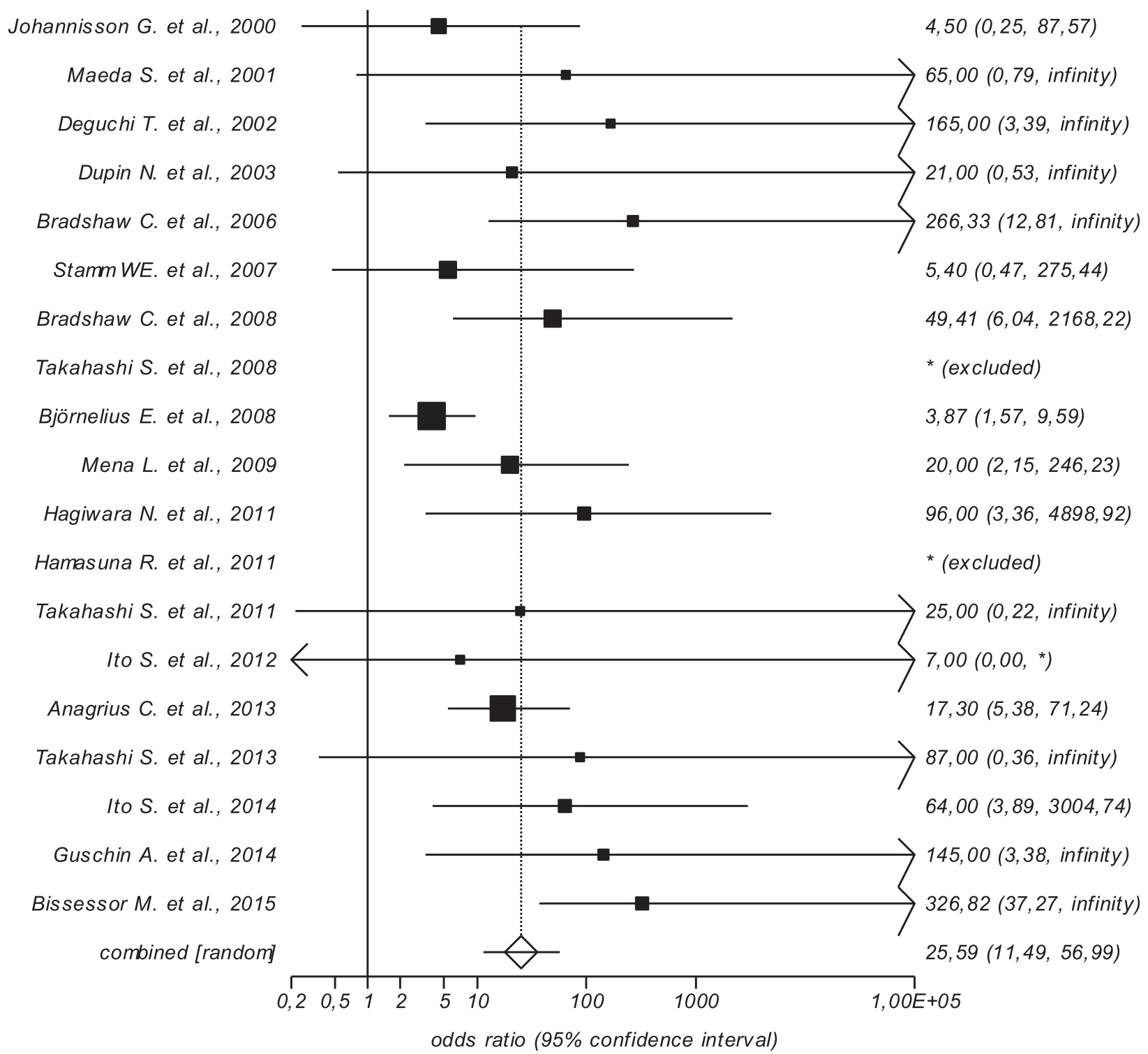

Fig. 1 Meta-analysis of the risk of persistence of urethritis signs and/or symptoms in patients with and without eradication of Mycoplasma genitalium. Data from 19 studies included; in two studies, OR could not be calculated due to eradication of $M$. genitalium in all patients 


\section{Antimicrobial treatment of $M$. genitalium}

Similar to other mycoplasmas, $M$. genitalium lacks a rigid peptidoglycan containing cell wall [43] and, consequently, $\beta$-lactam antibiotics and other antibiotics targeting the cell wall are not active. Determining the spectrum of antimicrobial susceptibility in vitro has been hampered by the limited number of strains capable of growing well enough in mycoplasma broth or agar to enable determination of the minimal inhibitory concentration (MIC) by standard Clinical and Laboratory Standards (CLSI) approved methods [44]. However, it has been shown that using susceptibility testing based on antimicrobial growth inhibition of M. genitalium in Vero cell culture, provides similar results to those obtained by conventional methods $[45,46]$, and, consequently, larger collections including fastidious strains could be tested [47].

Early in vitro studies with few strains showed that M. genitalium was highly susceptible to tetracyclines and macrolides, particularly to azithromycin, but that it had reduced susceptibility to older quinolones such as ofloxacin and ciprofloxacin [48]. When more strains were studied, it became clear that some strains were resistant to the older quinolones with MIC90 (the MIC covering $90 \%$ of the tested strains) for ciprofloxacin at $8 \mathrm{mg} / \mathrm{l}$. However, all of these strains were susceptible to moxifloxacin and other quinolones with extended Gram-positive spectrum such as sitafloxacin, sparfloxacin, and gatifloxacin $[46,49]$. With the emergence of resistance to both macrolides and quinolones as described below, the need for contemporary and representative strain collections has become evident in the search of new treatment modalities.

\section{Clinical efficacy of commonly used antimicrobials Doxycycline}

In vitro susceptibility testing suggests that most $M$. genitalium strains would be susceptible to doxycycline with a $\mathrm{MIC}_{50}$ of $0.25 \mathrm{mg} / \mathrm{l}$ and a $\mathrm{MIC}_{90}$ of $1 \mathrm{mg} / \mathrm{l}$ among 39 strains examined [47]. This is in striking contrast to clinical experience that predominantly showed poor efficacy of doxycycline in eradication of $M$. genitalium [50]. A single exception is an early study where 33 (94\%) of 35 men were $M$. genitalium negative after doxycycline treatment [51]. It should be noted, however, that eradication was evaluated only one week after treatment, and may reflect temporary suppression of the M. genitalium load, which has been reported in a study by Mena et al. [31] where $47 \%$ of men with early clinical cure after doxycycline experienced a subsequent relapse. In controlled clinical trials, the microbiological cure rate has ranged between $22 \%$ and $45 \%$ [30, 31, $52,53]$. The reason for the discrepancy between in vitro and in vivo activity is not clear, but at least two studies $[30,38]$ have found that eradication rates in women were slightly higher than in men (37\% vs $17 \%$, and $48 \%$ vs $38 \%$, respectively). Whether this is a reflection of lower compliance in men in relation to the nine-day doxycycline regimen used in these studies, or of an inaccessible prostatic focus of $M$. genitalium, remains speculative. However, based on these data doxycycline cannot be recommended for first line treatment of M. genitalium infection.

\section{Azithromycin}

In published studies, the majority of $M$. genitalium infected patients have been treated with azithromycin, and in early susceptibility studies [48], this macrolide was shown to be very potent. In patients with STIs, treatment with a $1 \mathrm{~g}$ single dose of azithromycin was well documented to be active in eradication of C. trachomatis [54], and the idea of a single-dose treatment was appealing for STI patients and widely adopted in many nations as first line treatment for NGU and M. genitalium. However, in M. pneumoniae pneumonia an extended treatment of $500 \mathrm{mg}$ day one followed by $250 \mathrm{mg}$ once daily on days two to five (referred to subsequently as extended azithromycin) was shown to be as effective as erythromycin for ten days [55]. Based on early, unpublished observations regarding the poor efficacy of doxycycline, Scandinavian researchers agreed that a slow-growing bacterium such as M. genitalium would need treatment for an extended period. Following the reported effect of extended azithromycin on the closely related $M$. pneumoniae [55], and approval of this regimen for treatment of pneumonia from the regulatory bodies, it was decided that this 5 day extended azithromycin regimen should be the preferred treatment for $M$. genitalium infections in Denmark, Norway and Sweden. A number of other extended regimens were considered, including giving a $1 \mathrm{~g}$ dose on day one in order to comply with treatment guidelines for C. trachomatis, and then supplementing with $250 \mathrm{mg}$ on days two to five. This regimen may have increased the acceptance of the idea of extended treatment among physicians treating STIs, and would not be expected to have adverse influence on the treatment of M. genitalium. However, eradication of $C$. trachomatis infections with the 5 day extended azithromycin regimen has recently been documented [56].

Over the years, controversy has existed over the optimal dosage of azithromycin [57]. Importantly, no randomised controlled trial has compared azithromycin $1 \mathrm{~g}$ single dose with extended azithromycin, but a few observational studies and one treatment trial [30, 38, 58, 59] have included patients treated with both regimens. In the four studies, 469 M. genitalium infected patients received azithromycin as a $1 \mathrm{~g}$ single dose, and 244 received extended azithromycin with microbiological cure rates of 81 and $88 \%$, respectively $(p=0.026)$. It should be noted, however, that a large proportion of the patients receiving the extended azithromycin had it as a second line treatment, most often after doxycycline, impacting on direct comparison of the 
two azithromycin regimens. One of the emerging concerns with $M$. genitalium is that the $1 \mathrm{~g}$ single dose may be more likely to select for macrolide resistance compared to the extended regimen [57]. No data from controlled trials are available, and only a single observational study [38] has examined the development of resistance after extended azithromycin. This study found that none of 25 patients $(0 \%$ [95 \% CI 0-13\%]) treated with extended azithromycin first line developed resistance, and, similarly, none (0\% [95\% CI 0-7 \%]) of 52 patients who received extended azithromycin second line after doxycycline developed macrolide resistance. This contrasts with the risk of development of post-treatment resistance reported among 318 patients treated with a $1 \mathrm{~g}$ azithromycin in six studies, which was $10 \%$ (95 \% CI 7-14 \%) [27, 38, 41, 60-62]. The $7 \%$ difference in microbiological cure rate between $1 \mathrm{~g}$ single dose and extended azithromycin regimens calculated above is strikingly similar to the $10 \%$ risk of development of posttreatment resistance after $1 \mathrm{~g}$ single dose azithromycin, lending support to the concept that single dose therapy appears to be associated with selection of resistance compared to extended regimens. The likely consequence of such rapid selection of resistant mutants without an efficient method to remove them from the population, is escalating macrolide resistance in high-risk populations. This situation appears to be playing out in countries where the use of $1 \mathrm{~g}$ azithromycin is widespread for chlamydia and its associated syndromes, and testing for M. genitalium is often restricted to speciality services or unavailable. Data from some of these nations, including in Greenland [63], Australia [60], Japan [64] and the UK [65], indicate macrolide resistance mutations are now detected in 30-100\% of cases with M. genitalium.

Macrolide resistance in $M$. genitalium is primarily caused by mutations in nucleotide 2058 or 2059 (Escherichia coli numbering) in region $\mathrm{V}$ of the $23 \mathrm{~S}$ rRNA gene [66] although mutations in position 2062 have been described after treatment with josamycin in vivo [42] and in M. pneumoniae by in vitro selection [67]. Analogous to M. pneumoniae, it would be expected however, that strains with mutations in position 2062 would retain susceptibility to azithromycin. M. genitalium has only one ribosomal RNA operon, and as mycoplasmas have a high mutation rate [68], single nucleotide changes that confer high-level antimicrobial resistance would also be likely to be randomly present in a population of $M$. genitalium cells (heterotypic resistance). This could explain the recent observed association in three studies between a high organism load and increased risk of treatment failure following both azithromycin and josamycin [41, 42, 62]. Bissessor et al. reported in men with urethral $M$. genitalium that for each $\log 10$ increase in organism load there was a significant increase in the odds of $1 \mathrm{~g}$ azithromycin failure (adjusted OR, 1.8, $p=.018$ ) [41]. Macrolides are bacteriostatic, and it is therefore possible that a high initial organism load could lead to a larger number of organisms surviving the initial peak concentrations of azithromycin, with replication of the surviving cells ensuing when concentrations drop below the MIC leading to a situation where spontaneously occurring mutations would be readily selected. Overall, the body of evidence suggests that both heterotypic resistance with a minority population of resistant strains being present before initiation of treatment and resistance developing during treatment are likely to play a role in macrolide failure, with peak concentration and duration of antibiotic exposure, together with organism load, contributing to the survival and emergence of resistant mutants.

Cure rates for $M$. genitalium following $1 \mathrm{~g}$ azithromycin appear to be declining internationally, with evidence of a lower cure rate in studies where patients were recruited most recently. In Fig. 2, 19 studies were stratified according to the mid-date of the reported patient inclusion. In the seven studies recruiting patients in the first half of the observation period before December 2005 [10, 28-31, 51, 69], 222 (87\%) of 255 patients were microbiologically cured. In contrast, for the 12 studies with mid-enrolment after November 2005 [22, 32, 39, 41, 52, 53, 58-62, 70], only 660

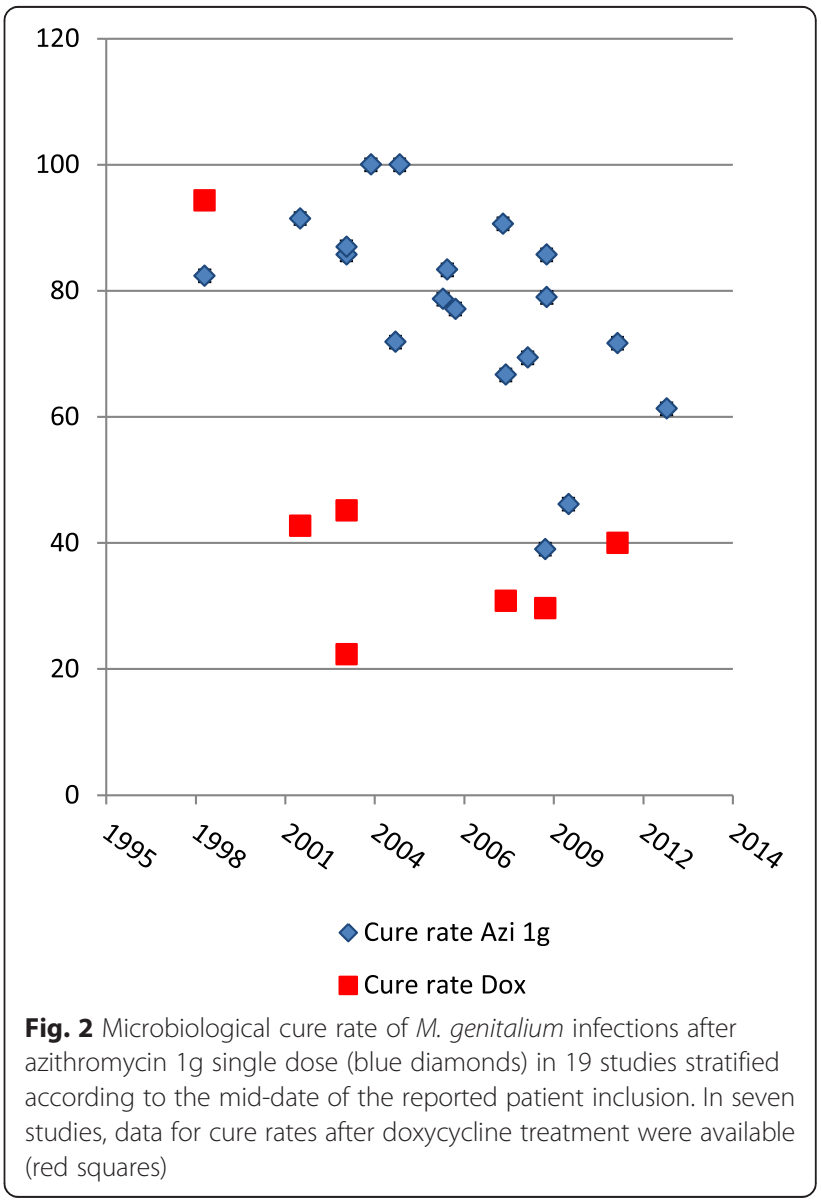


(71\%) of 925 patients were cured $(p<0.0001)$. Seven of the studies also presented data on doxycycline treatment, and apart from the early study by Gambini et al. [51], cure rates were low, between 22 and $45 \%$ (Fig. 2) but without an evident time trend.

\section{Quinolones}

Quinolones have been used in the treatment of $M$. genitalium, but based on in vitro susceptibility studies, it was predicted that second generation fluoroquinolones (ciprofloxacin and ofloxacin) would be inefficient in eradicating $M$. genitalium and this was confirmed in observational studies $[23,58]$ with an overall cure rate of $59 \%$. From in vitro data [46], the third generation quinolone levofloxacin appeared more promising, but in observational studies, again it only achieved a cure rate of $54 \%$ in 82 treated patients [24, 34, 70, 71]

Moxifloxacin is a fourth generation quinolone and it has been one of the most commonly used second line antimicrobials being reported first in 2006 [27], although it had been used several years before. Moxifloxacin is bactericidal, and generally well tolerated, and in early studies, it appeared to have a cure rate approaching $100 \%$ $[22,27,38,58]$. However, warnings of possible hepatotoxicity were added to the US labelling in 2007, and in 2008, moxifloxacin was restricted to second line indications in Europe [72]. However, these serious adverse events were subsequently shown to be no more common in patients treated with moxifloxacin as monotherapy, than in patients treated with amoxicillin or with doxycycline [72]. Aside from concerns of adverse events, a declining cure rate for moxifloxacin has now been observed, primarily in patients from the Asia-Pacific region. Treatment failures have recently been reported in up to $30 \%$ of patients treated with moxifloxacin, and a significant proportion of these patients' strains had concurrent macrolide resistance mediating mutation leaving very few available treatment options [41, 59, 61, 70]. Resistance to moxifloxacin and other fourth generation quinolones is mediated by mutations in quinolone resistance determining regions (QRDR) of the parC gene, primarily in the hotspots in amino acid positions S83 and D87 (M. genitalium numbering) [73, 74] reported also in $M$. pneumoniae, $M$. hominis and $U$. urealyticum [75-77]. Based on data on in vitro selection of resistance in $M$. pneumoniae, supporting mutations in gyrB or parE may also be needed to reach resistant MIC levels [77], but this has not yet been definitely determined for M. genitalium. Mutations in the relevant positions have been found at a low rate in Europe with only 1 (5\%) of 22 samples from London, UK [65] and even lower than this in Denmark (Jensen, unpublished). In the AsiaPacific region, however, evidence of fluoroquinolone mutations and associated treatment failure is emerging. In an Australian STI clinic $15 \%$ of 143 specimens collected between 2008 and 2011, carried mutations that were associated with fluoroquinolone resistance [78]. Similarly, a report from Japan [64] found parC mutations in 17 (33\%) of 51 specimens collected between 2011 and 2013, with a dramatic increase from $20 \%$ in 2011 to $47 \%$ in 2013. It is important to note, however, that the in vitro and in vivo correlates of mutations in the QRDR for several of the reported mutations have not yet been established. The increasing trend of parC mutations reported from Japan was mainly due to an S83N mutation [64], and this substitution between two polar amino acids with uncharged side-chains has been shown not to change the moxifloxacin MIC from that of the susceptible wild-type G37 $M$. genitalium type-strain (Jensen, unpublished). This observation probably explains the remarkable $100 \%$ cure-rate of sitafloxacin in nine $M$. genitalium infected patients with mutations in the QRDR of parC, eight of whom carried the indifferent S83N mutation.

Other fourth generation quinolones have been used in Japan. Gatifloxacin (now withdrawn from the market) cured $90 \%$ of 48 patients $[33,71]$ and sitafloxacin has been used in five studies $[36,37,39,64,70]$ with cure rates ranging from $100 \%$ to $85 \%$ with an overall cure rate of $95 \%$ in 105 patients. In vitro, sitafloxacin also appear to have a good activity with MICs for moxifloxacin susceptible strains approximately one dilution step below that of moxifloxacin [46]. For moxifloxacin resistant strains, the MIC is currently being examined, but for one moxifloxacin resistant strain, an encouraging MIC of $1 \mathrm{mg} / \mathrm{l}$ has been found (R. Hamasuna, personal communication).

\section{Other licensed but less commonly used antibiotics}

Pristinamycin Pristinamycin is an oral streptogramin antibiotic with bactericidal activity against Gram-positive organisms including methicillin-resistant Staphylococcus aureus (MRSA). It has a high activity against macrolide susceptible $M$. genitalium strains [48] and even for strains with combined macrolide and moxifloxacin resistance, the MIC remained $<1 \mathrm{mg} / \mathrm{l}$ (Jensen, unpublished). Pristinamycin has been used for decades, particularly in France, for treatment of MRSA, but is not registered outside of France and some North-African countries. In M. pneumoniae, it has a relatively lower risk of development of resistance than azithromycin in vitro, and the selected mutants retained an azithromycin susceptible phenotype [67]. How a strain with pre-existing azithromycin resistance would react to selection with pristinamycin remains unknown, but in vitro selection studies are currently ongoing. Pristinamycin has been successfully used as third line treatment for patients infected with multidrug resistant M. genitalium strains. As most of these patients were facing their last known active antimicrobial therapy, the maximal recommended dose of $1 \mathrm{~g}$ four times orally a day for 10 days was used. Several patients in Scandinavia have responded well 
to this treatment, and recently a case series from Australia demonstrated six cases with successful eradication [41]. Using pristinamycin as a second line antibiotic instead of moxifloxacin would probably be hampered both by the price of pristinamycin and of the compliance issues associated with four times a day dosage. Whether lower daily doses would be effective remains to be determined, but for patients failing azithromycin, moxifloxacin, and possibly 14 days of doxycycline, a dose reduction is not advisable due to the proportion of these multidrug resistant strains that have an elevated MIC of $0.5 \mathrm{mg} / \mathrm{l}$ (Jensen, unpublished).

\section{Antimicrobials under development}

Solithromycin is a newly developed fluoroketolide (CEM101), a further development of the macrolides. It has a potent activity against azithromycin susceptible $M$. genitalium strains and to some extent also against azithromycin resistant strains [47]. Susceptibility to solithromycin appears to differ according to the type of macrolide resistance mediating mutation present, with eight strains harbouring mutations in position A2059 having susceptible MICs $<2 \mathrm{mg} / \mathrm{l}$, but only two of five strains with the A2058G mutation with susceptible MICs [47]. Using a clinical scenario seen in Denmark where $60 \%$ of azithromycin resistant strains carry the A2058G mutation [79], one would expect to achieve a $65 \%$ cure rate with the use of solithromycin for azithromycin resistant strains [47]. Treatment trials of single dose solithromycin for patients with gonorrhoea are currently underway, and in one study, a few cases of concomitant $M$. genitalium infections have been detected of which some have been eradicated [80]; however, data on the efficacy of solithromycin for $M$. genitalium is limited. Treatment trials targeting patients with NGU are likely to need a longer treatment duration in order to cover M. genitalium infections.

Lefamulin (BC-3781) belongs to the pleuromutilin class of antimicrobials, which has been used for decades in the veterinary industry for infections in pigs, and to a lesser extent in poultry, but this class has not previously been developed for human use. Lefamulin has a very potent activity in vitro with $\mathrm{MIC} \leq 0.06 \mathrm{mg} / \mathrm{l}$ against multidrug resistant $M$. genitalium strains [81]. In the veterinary mycoplasmas, mutations in position A2058 have been associated with increased MIC levels, but only in a background of other mutations in the 23S rRNA gene, suggesting multistep development of significant resistance [82]. Lefamulin has successfully completed phase II trials for skin and soft tissue infections [83] and is currently entering trials for community-acquired pneumonia. It has not been studied in the treatment of STIs or specifically M. genitalium.

A novel spiropyrimidinetrione AZD0914 (a DNA gyrase inhibitor) has been tested against azithromycin and quinolone susceptible strains of M. genitalium and MIC
$<1 \mathrm{mg} / \mathrm{l}$ was reported for 11 strains [84]. No reports on human use of this interesting class of compounds have been published.

LBM415 belongs to a new antimicrobial class, the peptide deformylase inhibitors which inhibit bacterial protein synthesis. The compound has high in vitro activity against $M$. pneumoniae and was efficient in eradicating the bacterium in a mouse model [85], but unfortunately, prolonged high dosage produced methaemoglobulinaemia [86] and further development of the compound has apparently been stopped.

\section{Possible use of other registered antimicrobials}

Although tetracyclines appear to be active in vitro, they have never shown evidence of sufficient efficacy clinically. Whether newer modifications of the tetracyclines such as the glycylcycline tigecycline would be active in M. genitalium infections remain to be determined. This antibiotic is active in vitro against $M$. pneumoniae [87], but at present, no in vitro data are available for $M$. genitalium, and the need for parenteral administration would not render it an optimal treatment, except in otherwise untreatable infections. Linezolid with its Gram-positive spectrum does not appear promising according to the MICs obtained for M. pneumoniae [87], but it has not been evaluated for M. genitalium.

Chloramphenicol has a relatively high MIC in vitro $(5-25 \mathrm{mg} / \mathrm{ml})$ [49] and although organisms with an MIC $\leq 16 \mathrm{mg} / \mathrm{l}$ are considered susceptible, is not likely to be a promising alternative. A derivative of chloramphenicol, thiamphenicol, has been used in treatment of NGU [88] and PID [89], and is reported to have a higher activity than chloramphenicol against mycoplasmas. It is currently registered in Italy and Brazil. Spectinomycin is widely used in the veterinary industry to treat mycoplasmal infections, however, most often in combination with lincomycin, probably to reduce the rapid development of resistance and due to a synergistic effect. The spectinomycin MIC breakpoint for $N$. gonorrhoeae is $32-64 \mathrm{mg} / \mathrm{l}$ and the MIC for M. genitalium has been reported to be $<25 \mathrm{mg} / \mathrm{l}$ [49]. Since spectinomycin is inactive against $C$. trachomatis but has some activity against ureaplasmas, it has been used in the past to substantiate the role of ureaplasmas in NGU [90]. Spectinomycin may have a potential role as a treatment option for multidrug resistant strains of $M$. genitalium but the need for parenteral administration would be problematic. Lastly, successful eradication of ureaplasmas has been reported in a hypogammaglobulinaemic patient with untreatable urethritis after treatment with netilmicin [91]. However, aminoglycosides would not be expected to be active in vivo against $M$. genitalium, as even for the most potent aminoglycoside, netilmicin, the MIC of some M. genitalium strains was $25 \mathrm{mg} / \mathrm{l}$ [49]. Furthermore, as M. genitalium is capable of surviving intracellularly 
[92, 93], aminoglycosides would not be optimal as their intracellular concentration is very low.

\section{Conclusions}

$M$. genitalium has shown a remarkable capability to develop antimicrobial resistance very rapidly after introduction of new treatment modalities. It has already become a difficult bacterium to treat on a syndromic basis, and even after specific detection, enormous local differences exist in the prevalence of strains with macrolide resistancemediating mutations, highlighting the need for widespread antimicrobial resistance surveillance. In the ideal clinical setting, specific diagnostic tests for M. genitalium would be as readily available as tests for $C$. trachomatis and $N$. gonorrhoeae, and detection of M. genitalium would be accompanied or followed by molecular detection of macrolide resistance mediating mutations. For patients infected with a macrolide susceptible strain, evidence suggests that extended azithromycin should be the first-line treatment, but a test of cure should be routinely performed at three to four weeks, as resistance may develop even with the extended regimen. Patients with macrolide resistance can in most settings still be treated with moxifloxacin $400 \mathrm{mg}$ once daily for 7-10 days, but treatment failure can occur, particularly in the Asia-Pacific region, where pre-existing quinolone resistance is common and, therefore, a test of cure 3-4 weeks after treatment is again highly recommended. If a patient experiences failure of azithromycin and moxifloxacin in the absence of reinfection, whether doxycycline $100 \mathrm{mg}$ twice daily for 14 days should be attempted before pristinamycin is not clear, but the former is more readily available in most clinical settings. Most patients responding to doxycycline will have a negative test of cure by the end of treatment [94], but if they are still positive, treatment with pristinamycin $1 \mathrm{~g}$ four times daily for 10 days is currently the only third or fourth line antimicrobial treatment that is available. The important question is what to do when this treatment fails, which has been experienced by a limited number of patients but will inevitably increase in the foreseeable future. Based on the rapid emergence of resistance to first and second line treatment options internationally, continuous monitoring of the efficacy of treatment regimens for $M$. genitalium is highly recommended in clinical settings. In patients failing treatment, samples appropriate for culture should ideally be collected in order both to isolate M. genitalium for confirmation of the in-vitro correlates of antimicrobial resistance, and to elucidate the molecular mechanisms leading to treatment failure.

In clinical settings where access to testing for M. genitalium is not currently available, consideration should be given to changing presumptive treatment of NGU and cervicitis from the current recommendation of $1 \mathrm{~g}$ azithromycin to doxycycline in order to minimise the development and escalation of macrolide resistance. Patients failing doxycycline could then be subsequently treated with extended azithromycin, or in settings where macrolide resistance is already widespread, with moxifloxacin, if available. Access to M. genitalium testing in patients failing secondary treatment is available in many countries or via international collaborations, and should be used in these specific cases in order to optimise patient management.

Future research should focus on in vitro evaluation and subsequent treatment trials of untested, but registered antimicrobials, and emphasis should be on the development of treatment algorithms including dual therapy and resistance testing in order to minimise the development of resistance. Such combination therapies will need to be rapidly adopted and accompanied by antimicrobial resistance surveillance in order to retain susceptibility. As it has been the case for $N$. gonorrhoeae, the rapid development of resistance in M. genitalium highlights the fact that the era of single dose therapy for uncomplicated STIs is long past. Combination therapy and resistance testing together with the development and evaluation of new classes of antimicrobials should enable us to finally hit this fast moving target.

\section{Competing interests}

JSJ has no personal competing interests, however, Statens Serum Institut has received remuneration for contract work regarding antimicrobial susceptibility testing from Cempra (solithromycin) and Nabriva (Lefamulin) and other compounds not mentioned in this review. Statens Serum Institut performs commercial testing for M. genitalium and for antimicrobial resistance mediating mutations using molecular methods. CB has no competing interests.

\section{Authors' contributions}

JSJ collected data from published studies and carried out the metaanalyses. JSJ produced the first draft of the manuscript. CB critically reviewed the manuscript, added clinically relevant information and provided input in the planning phase of the review. Both authors read and approved the final manuscript.

\section{Acknowledgements}

Lars Falk and Ryoichi Hamasuna are thanked for sharing unpublished data. Alexander Guschin and Zarin Gundevia are thanked for responding to emails clarifying data on treatment efficacy.

\section{Author details}

${ }^{1}$ Microbiology and Infection Control, Sexually Transmitted Bacterial Infections, Research and Development, Statens Serum Institut, Artillerivej 5, Copenhagen DK-2300, Denmark. ${ }^{2}$ Central Clinical School. Monash University, Melbourne, VIC, Australia. ${ }^{3}$ Melbourne Sexual Health Centre, The Alfred Hospital, Melbourne, VIC, Australia.

Received: 21 May 2015 Accepted: 20 July 2015

Published online: 19 August 2015

\section{References}

1. Tully JG, Taylor Robinson D, Cole RM, Rose DL. A newly discovered mycoplasma in the human urogenital tract. Lancet. 1981;:1:1288-91.

2. Jensen JS, Uldum SA, Søndergård-Andersen J, Vuust J, Lind K. Polymerase chain reaction for detection of Mycoplasma genitalium in clinical samples. J Clin Microbiol. 1991;29:46-50.

3. Palmer HM, Gilroy CB, Furr PM, Taylor-Robinson D. Development and evaluation of the polymerase chain reaction to detect Mycoplasma genitalium. FEMS Microbiol Lett. 1991;61:199-203. 
4. Jensen JS, Ørsum R, Dohn B, Uldum S, Worm AM, Lind K. Mycoplasma genitalium: A cause of male urethritis? Genitourin Med. 1993;69:265-9.

5. Horner PJ, Gilroy CB, Thomas BJ, Naidoo RO, Taylor-Robinson D. Association of Mycoplasma genitalium with acute non-gonococcal urethritis. Lancet. 1993:342:582-5.

6. Taylor-Robinson D, Jensen JS. Mycoplasma genitalium: from chrysalis to multicolored butterfly. Clin Microbiol Rev. 2011;24:498-514.

7. Daley G, Russell D, Tabrizi S, McBride J. Mycoplasma genitalium: a review. Int J STD AIDS. 2014;25:475-87.

8. Cohen CR, Manhart LE, Bukusi EA, Astete S, Brunham RC, Holmes KK, et al. Association between Mycoplasma genitalium and acute endometritis. Lancet. 2002;359:765-6.

9. Manhart LE, Critchlow CW, Holmes KK, Dutro SM, Eschenbach DA, Stevens CE, et al. Mucopurulent cervicitis and Mycoplasma genitalium. J Infect Dis. 2003;187:650-7.

10. Anagrius C, Loré B, Jensen JS. Mycoplasma genitalium: prevalence, clinical significance, and transmission. Sex Transm Infect. 2005;81:458-62.

11. Falk $L$, Fredlund $H$, Jensen JS. Signs and symptoms of urethritis and cervicitis among women with or without Mycoplasma genitalium or Chlamydia trachomatis infection. Sex Transm Infect. 2005;81:73-8.

12. Lis R, Rowhani Rahbar A, Manhart LE. Mycoplasma genitalium infection and female reproductive tract disease: A meta-analysis. Clin Infect Dis. 2015;6(3):418-26.

13. Oakeshott P, Hay P, Taylor-Robinson D, Hay S, Dohn B, Kerry S, et al. Prevalence of Mycoplasma genitalium in early pregnancy and relationship between its presence and pregnancy outcome. BJOG. 2004;111:1464-7.

14. Falk L, Fredlund $H$, Jensen JS. Symptomatic urethritis is more prevalent in men infected with Mycoplasma genitalium than with Chlamydia trachomatis. Sex Transm Infect. 2004;80:289-93.

15. Björnelius $E$, Lidbrink $P$, Jensen JS. Mycoplasma genitalium in non-gonococcal urethritis - a study in Swedish male STD patients. Int J STD AIDS. 2000;11:292-6.

16. Horner PJ, Taylor-Robinson D. Mycoplasma genitalium and non-gonococcal urethritis. Lancet. 1994;343:790-1.

17. Bradshaw CS, Tabrizi SN, Read TR, Garland SM, Hopkins CA, Moss LM, et al. Etiologies of nongonococcal urethritis: bacteria, viruses, and the association with orogenital exposure. J Infect Dis. 2006;193:336-45.

18. Andersen B, Sokolowski I, Østergaard L, Moller JK, Olesen F, Jensen JS. Mycoplasma genitalium: prevalence and behavioural risk factors in the general population. Sex Transm Infect. 2007:83:237-41.

19. Oakeshott P, Aghaizu A, Hay P, Reid F, Kerry S, Atherton H, et al. Is Mycoplasma genitalium in women the "New Chlamydia?" A communitybased prospective cohort study. Clin Infect Dis. 2010;51:1160-6.

20. Manhart LE, Holmes KK, Hughes JP, Houston LS, Totten PA. Mycoplasma genitalium among young adults in the United States: an emerging sexually transmitted infection. Am J Public Health. 2007;97:1118-25.

21. Wikström A, Jensen JS. Mycoplasma genitalium: a common cause of persistent urethritis among men treated with doxycycline. Sex Transm Infect. 2006;82:276-9.

22. Bradshaw CS, Chen MY, Fairley CK. Persistence of Mycoplasma genitalium following azithromycin therapy. PLoS One. 2008;3:e3618.

23. Johannisson $G$, Enström $Y$, Löwhagen GB, Nagy $V$, Ryberg K, Seeberg S, et al. Occurrence and treatment of Mycoplasma genitalium in patients visiting STD clinics in Sweden. Int J STD AIDS. 2000;11:324-6.

24. Maeda SI, Tamaki M, Kojima K, Yoshida T, Ishiko H, Yasuda M, et al. Association of Mycoplasma genitalium persistence in the urethra with recurrence of nongonococcal urethritis. Sex Transm Dis. 2001;28:472-6.

25. Deguchi T, Yoshida T, Yokoi S, Ito M, Tamaki M, Ishiko H, et al. Longitudinal quantitative detection by real-time PCR of Mycoplasma genitalium in firstpass urine of men with recurrent nongonococcal urethritis. J Clin Microbiol. 2002:40:3854-6.

26. Dupin N, Bijaoui G, Schwarzinger M, Ernault P, Gerhardt P, Jdid R, et al. Detection and quantification of Mycoplasma genitalium in male patients with urethritis. Clin Infect Dis. 2003;37:602-5.

27. Bradshaw CS, Jensen JS, Tabrizi SN, Read TR, Garland SM, Hopkins CA, et al. Azithromycin failure in Mycoplasma genitalium urethritis. Emerg Infect Dis. 2006;12:1149-52

28. Stamm WE, Batteiger BE, McCormack WM, Totten PA, Sternlicht A, Kivel NM. A randomized, double-blind study comparing single-dose rifalazil with single-dose azithromycin for the empirical treatment of nongonococcal urethritis in men. Sex Transm Dis. 2007;34:545-52.

29. Takahashi S, Matsukawa M, Kurimura Y, Takeyama K, Kunishima Y, Iwasawa A, et al. Clinical efficacy of azithromycin for male nongonococcal urethritis. J Infect Chemother. 2008;14:409-12.
30. Björnelius E, Anagrius C, Bojs G, Carlberg H, Johannisson G, Johansson E, et al. Antibiotic treatment of symptomatic Mycoplasma genitalium infection in Scandinavia: a controlled clinical trial. Sex Transm Infect. 2008;84:72-6.

31. Mena LA, Mroczkowski TF, Nsuami M, Martin DH. A randomized comparison of azithromycin and doxycycline for the treatment of Mycoplasma genitalium-positive urethritis in men. Clin Infect Dis. 2009;48:1649-54.

32. Hagiwara N, Yasuda M, Maeda S, Deguchi T. In vitro activity of azithromycin against Mycoplasma genitalium and its efficacy in the treatment of male Mycoplasma genitalium-positive nongonococcal urethritis. J Infect Chemother. 2011;17:821-4.

33. Hamasuna R, Takahashi S, Kiyota H, Yasuda M, Hayami H, Arakawa S, et al. Effect of gatifloxacin against Mycoplasma genitalium-related urethritis: an open clinical trial. Sex Transm Infect. 2011;87:389-90.

34. Takahashi S, Ichihara K, Hashimoto J, Kurimura Y, Iwasawa A, Hayashi K, et al. Clinical efficacy of levofloxacin $500 \mathrm{mg}$ once daily for 7 days for patients with non-gonococcal urethritis. J Infect Chemother. 2011;17:392-6.

35. Sena AC, Lensing S, Rompalo A, Taylor SN, Martin DH, Lopez LM, et al. Chlamydia trachomatis, Mycoplasma genitalium, and Trichomonas vaginalis infections in men with nongonococcal urethritis: predictors and persistence after therapy. J Infect Dis. 2012;206:357-65.

36. Ito S, Yasuda M, Seike K, Sugawara T, Tsuchiya T, Yokoi S, et al. Clinical and microbiological outcomes in treatment of men with non-gonococcal urethritis with a 100-mg twice-daily dose regimen of sitafloxacin. J Infect Chemother. 2012;18:414-8.

37. Takahashi S, Hamasuna R, Yasuda M, Ito S, Ito K, Kawai S, et al. Clinical efficacy of sitafloxacin $100 \mathrm{mg}$ twice daily for 7 days for patients with non-gonococcal urethritis. J Infect Chemother. 2013;19:941-5.

38. Anagrius C, Lore B, Jensen JS. Treatment of Mycoplasma genitalium. Observations from a Swedish STD Clinic. PLOS ONE. 2013;8:e61481.

39. Ito S, Mizutani K, Seike K, Sugawara T, Tsuchiya T, Yasuda M, et al. Prediction of the persistence of Mycoplasma genitalium after antimicrobial chemotherapy by quantification of leukocytes in first-void urine from patients with non-gonococcal urethritis. J Infect Chemother. 2014;20:298-302.

40. Takahashi S, Kiyota H, Ito S, Iwasawa A, Hiyama Y, Uehara T, et al. Clinical efficacy of a single two gram dose of azithromycin extended release for male patients with urethritis. Antibiotics. 2014;3:02.

41. Bissessor M, Tabrizi SN, Twin J, Abdo H, Fairley CK, Chen MY, et al. Macrolide resistance and azithromycin failure in a Mycoplasma genitalium-infected cohort and response of azithromycin failures to alternative antibiotic regimens. Clin Infect Dis. 2015;60:1228-36.

42. Guschin A, Ryzhikh P, Rumyantseva T, Gomberg M, Unemo M. Treatment efficacy, treatment failures and selection of macrolide resistance in patients with high load of Mycoplasma genitalium during treatment of male urethritis with josamycin. BMC Infect Dis. 2015;15:40.

43. Taylor-Robinson D, Gilroy CB, Jensen JS. The biology of Mycoplasma genitalium. Venereol. 2000;13:119-27.

44. Waites KB, Duffy LB, Bebear CM, Matlow A, Talkington DF, Kenny GE, et al. Standardized methods and quality control limits for agar and broth microdilution susceptibility testing of Mycoplasma pneumoniae, Mycoplasma hominis, and Ureaplasma urealyticum. J Clin Microbiol. 2012;50:3542-7.

45. Hamasuna R, Osada Y, Jensen JS. Antibiotic susceptibility testing of Mycoplasma genitalium by TaqMan 5' nuclease real-time PCR. Antimicrob Agents Chemother. 2005;49:4993-8.

46. Hamasuna R, Jensen JS, Osada Y. Antimicrobial susceptibilities of Mycoplasma genitalium by broth dilution and quantitative PCR. Antimicrob Agents Chemother. 2009;53:4938-9.

47. Jensen JS, Fernandes P, Unemo M. In vitro activity of the new fluoroketolide solithromycin (CEM-101) against macrolide-resistant and - susceptible Mycoplasma genitalium strains. Antimicrob Agents Chemother. 2014;58:3151-6

48. Renaudin H, Tully JG, Bebear C. In-vitro susceptibilities of Mycoplasma genitalium to antibiotics. Antimicrob Agents Chemother. 1992;36:870-2.

49. Hannan PC. Comparative susceptibilities of various AIDS-associated and human urogenital tract mycoplasmas and strains of Mycoplasma pneumoniae to 10 classes of antimicrobial agent in vitro. J Med Microbiol. 1998:47:1115-22.

50. Falk $L$, Fredlund $H$, Jensen JS. Tetracycline treatment does not eradicate Mycoplasma genitalium. Sex Transm Infect. 2003;79:318-9.

51. Gambini D, Decleva I, Lupica L, Ghislanzoni M, Cusini M, Alessi E. Mycoplasma genitalium in males with nongonococcal urethritis: prevalence and clinical efficacy of eradication. Sex Transm Dis. 2000;27:226-9. 
52. Schwebke JR, Rompalo A, Taylor S, Sena AC, Martin DH, Lopez LM, et al. Re-evaluating the treatment of nongonococcal urethritis: Emphasizing emerging pathogens - A Randomized Clinical Trial. Clin Infect Dis. 2011;52:163-70.

53. Manhart LE, Gillespie CW, Lowens MS, Khosropour CM, Colombara DV, Golden MR, et al. Standard treatment regimens for nongonococcal urethritis have similar but declining cure rates: a randomized controlled trial. Clin Infect Dis. 2013;56:934-42.

54. Martin DH, Mroczkowski TF, Dalu ZA, Mccarty J, Jones RB, Hopkins SJ, et al. A controlled trial of a single dose of azithromycin for the treatment of chlamydial urethritis and cervicitis. N Engl J Med. 1992;327:921-5.

55. Schönwald S, Gunjaca M, Kolacny Babic L, Car V, Gosev M. Comparison of azithromycin and erythromycin in the treatment of atypical pneumonias. J Antimicrob Chemother. 1990;25(Suppl A):123-6.

56. Unemo M, Endre KM, Moi H. Five-day azithromycin treatment regimen for mycoplasma genitalium infection also effectively eradicates chlamydia trachomatis. Acta Derm Venereol. 2015;95(6):730-2.

57. Horner P, Blee K, Adams E. Time to manage Mycoplasma genitalium as an STI: but not with azithromycin 1 g! Curr Opin Infect Dis. 2014;27:68-74.

58. Jernberg E, Moghaddam A, Moi H. Azithromycin and moxifloxacin for microbiological cure of Mycoplasma genitalium infection: an open study. Int J STD AIDS. 2008;19:676-9.

59. Gundevia Z, Foster R, Jamil MS, McNulty A. Positivity at test of cure following first-line treatment for genital Mycoplasma genitalium: follow-up of a clinical cohort. Sex Transm Infect. 2015;91:11-3.

60. Twin J, Jensen JS, Bradshaw CS, Garland SM, Fairley CK, Min LY, et al. Transmission and selection of macrolide resistant Mycoplasma genitalium infections detected by rapid high resolution melt analysis. PLoS One. 2012;7:e35593.

61. Couldwell DL, Tagg KA, Jeoffreys NJ, Gilbert GL. Failure of moxifloxacin treatment in Mycoplasma genitalium infections due to macrolide and fluoroquinolone resistance. Int J STD AIDS. 2013;24:822-8.

62. Walker J, Fairley CK, Bradshaw CS, Tabrizi SN, Twin J, Chen MY, et al. Mycoplasma genitalium incidence, organism load, and treatment failure in a cohort of young Australian women. Clin Infect Dis. 2013;56:1094-100.

63. Gesink DC, Mulvad G, Montgomery-Andersen R, Poppel U, MontgomeryAndersen S, Binzer A, et al. Mycoplasma genitalium presence, resistance and epidemiology in Greenland. Int J Circumpolar Health. 2012;11:1-8.

64. Kikuchi M, Ito S, Yasuda M, Tsuchiya T, Hatazaki K, Takanashi M, et al. Remarkable increase in fluoroquinolone-resistant Mycoplasma genitalium in Japan. J Antimicrob Chemother. 2014;69(9):2376-82.

65. Pond MJ, Nori AV, Witney AA, Lopeman RC, Butcher PD, Sadiq ST. High prevalence of antibiotic-resistant Mycoplasma genitalium in nongonococcal urethritis: the need for routine testing and the inadequacy of current treatment options. Clin Infect Dis. 2014;58:631-7.

66. Jensen JS, Bradshaw CS, Tabrizi SN, Fairley CK, Hamasuna R. Azithromycin treatment failure in Mycoplasma genitalium-positive patients with nongonococcal urethritis is associated with induced macrolide resistance. Clin Infect Dis. 2008:47:1546-53.

67. Pereyre S, Guyot C, Renaudin H, Charron A, Bebear C, Bebear CM. In vitro selection and characterization of resistance to macrolides and related antibiotics in Mycoplasma pneumoniae. Antimicrob Agents Chemother. 2004;48:460-5.

68. Woese CR, Stackebrandt E, Ludwig W. What are mycoplasmas: the relationship of tempo and mode in bacterial evolution. J Mol Evol. 1984;21:305-16.

69. Bradshaw CS, Tabrizi SM, Read TR, Hopkins CA, Moss MB, Garland SM, et al. Mycoplasma genitalium, Herpes Simplex viruses and adenoviruses in nongonococcal urethritis. International Society for Sexually Transmitted Diseases Research - Program and Book of Abstracts (16th ISSTDR meeting). 2005:70-71

70. Terada M, Izumi K, Ohki E, Yamagishi Y, Mikamo H. Antimicrobial efficacies of several antibiotics against uterine cervicitis caused by Mycoplasma genitalium. J Infect Chemother. 2012;18:313-7.

71. Yasuda M, Maeda S, Deguchi T. In vitro activity of fluoroquinolones against Mycoplasma genitalium and their bacteriological efficacy for treatment of $M$. genitalium-positive nongonococcal urethritis in men. Clin Infect Dis. 2005;41:1357-9.

72. Kaye JA, Castellsague J, Bui CL, Calingaert B, McQuay LJ, Riera-Guardia N, et al. Risk of acute liver injury associated with the use of moxifloxacin and other oral antimicrobials: a retrospective, population-based cohort study. Pharmacotherapy. 2014;34:336-49.

73. Shimada Y, Deguchi T, Nakane K, Masue T, Yasuda M, Yokoi S, et al. Emergence of clinical strains of Mycoplasma genitalium harbouring alterations in ParC associated with fluoroquinolone resistance. Int J Antimicrob Agents. 2010;36:255-8.
74. Deguchi T, Maeda S, Tamaki M, Yoshida T, Ishiko H, Ito M, et al. Analysis of the gyrA and parC genes of Mycoplasma genitalium detected in first-pass urine of men with non-gonococcal urethritis before and after fluoroquinolone treatment. J Antimicrob Chemother. 2001;48:742-4.

75. Bebear CM, Renaudin H, Charron A, Gruson D, Lefrancois M, Bebear C. In vitro activity of trovafloxacin compared to those of five antimicrobials against mycoplasmas including Mycoplasma hominis and Ureaplasma urealyticum fluoroquinolone-resistant isolates that have been genetically characterized. Antimicrob Agents Chemother. 2000;44:2557-60.

76. Bebear CM, Renaudin J, Charron A, Renaudin H, de Barbeyrac B, Schaeverbeke T, et al. Mutations in the gyrA, parC, and parE genes associated with fluoroquinolone resistance in clinical isolates of Mycoplasma hominis. Antimicrob Agents Chemother. 1999;43:954-6.

77. Gruson D, Pereyre S, Renaudin H, Charron A, Bebear C, Bebear CM. In vitro development of resistance to six and four fluoroquinolones in Mycoplasma pneumoniae and Mycoplasma hominis, respectively. Antimicrob Agents Chemother. 2005:49:1190-3.

78. Tagg KA, Jeoffreys NJ, Couldwell DL, Donald JA, Gilbert GL. Fluoroquinolone and macrolide resistance-associated mutations in Mycoplasma genitalium. J Clin Microbiol. 2013;51:2245-9.

79. Salado-Rasmussen K, Jensen JS. Mycoplasma genitalium testing pattern and macrolide resistance: A Danish nationwide retrospective survey. Clin Infect Dis. 2014:59:24-30.

80. Hook EW, III, Golden M, Jamieson BD, Dixon PB, Harbison HS, Lowens S et al:: A phase 2 trial of oral solithromycin $1200 \mathrm{mg}$ or $1000 \mathrm{mg}$ as single-dose oral therapy for uncomplicated gonorrhea. Clin Infect Dis 2015. Epub ahead of print

81. Paukner S, Gruss A, Fritsche TR, Ivezic-Schoenfeld Z, Jones RN. In vitro activity of the novel pleuromutilin BC-3781 tested against bacterial pathogens causing sexually transmitted diseases (STD). Interscience Conference of Antimicrobial Agents and Chemotherapy (ICAAC 2014) abstract. 2014:E-1183

82. Li BB, Shen JZ, Cao XY, Wang Y, Dai L, Huang SY, et al. Mutations in 23 S rRNA gene associated with decreased susceptibility to tiamulin and valnemulin in Mycoplasma gallisepticum. FEMS Microbiol Lett. 2010;308:144-9.

83. Prince WT, Ivezic-Schoenfeld Z, Lell C, Tack KJ, Novak R, Obermayr F, et al. Phase II clinical study of BC-3781, a pleuromutilin antibiotic, in treatment of patients with acute bacterial skin and skin structure infections. Antimicrob Agents Chemother. 2013;57:2087-94.

84. Waites KB, Crabb DM, Duffy LB, Huband MD. In vitro antibacterial activity of AZD0914 against human mycoplasmas and ureaplasmas. Antimicrob Agents Chemother. 2015;59(6):3627-9.

85. Fonseca-Aten M, Salvatore CM, Mejias A, Rios AM, Chavez-Bueno S, Katz K, et al. Evaluation of LBM415 (NVP PDF-713), a novel peptide deformylase inhibitor, for treatment of experimental Mycoplasma pneumoniae pneumonia. Antimicrob Agents Chemother. 2005;49:4128-36.

86. Rolan P, Sun H, Macleod C, Bracken K, Evans TG. Pharmacokinetics and unexpected safety issues of LBM415, a novel oral peptide deformylase inhibitor. Clin Pharmacol Ther. 2011;90:256-62.

87. Kenny GE, Cartwright FD. Susceptibilities of Mycoplasma hominis, M. pneumoniae, and Ureaplasma urealyticum to GAR-936, dalfopristin, dirithromycin, evernimicin, gatifloxacin, linezolid, moxifloxacin, quinupristin-dalfopristin, and telithromycin compared to their susceptibilities to reference macrolides, tetracyclines, and quinolones. Antimicrob Agents Chemother. 2001;45:2604-8.

88. Saito I. Clinical evaluation of thiamphenicol in treatment of nongonococcal urethritis. Sex Transm Dis. 1984;11:460-2.

89. Vige PM, Henrion RM. Thiamphenicol for treatment of salpingitis. Sex Transm Dis. 1984;11:441-3.

90. Bowie WR, Floyd JF, Miller Y, Alexander ER, Holmes J, Holmes KK. Differential response of chlamydial and ureaplasma-associated urethritis to sulphafurazole (sulfisoxazole) and aminocyclitols. Lancet. 1976;2:1276-8.

91. Taylor-Robinson D, Furr PM, Webster AD. Ureaplasma urealyticum causing persistent urethritis in a patient with hypogammaglobulinaemia. Genitourin Med. 1985;61:404-8.

92. Jensen JS, Blom J, Lind K. Intracellular location of Mycoplasma genitalium in cultured Vero cells as demonstrated by electron microscopy. Int J Exp Pathol. 1994;75:91-8.

93. Dallo SF, Baseman JB. Intracellular DNA replication and long-term survival of pathogenic mycoplasmas. Microb Pathog. 2000:29:301-9.

94. Falk L, Enger M, Jensen, JS. Time to eradication of Mycoplasma genitalium after antibiotic treatment in men and women. J Antimicrob Chemother in press. 2015. doi:10.1093/jac/dkv246 